\title{
Fast Error-Correcting Graph Isomorphism Based on Model Precompilation
}

\author{
B.T. Messmer and H. Bunke \\ Institut für Informatik und angewandte Mathematik, Universität Bern, \\ Neubrückstr. 10, Bern, Switzerland, bunke@iam.unibe.ch
}

\begin{abstract}
In this paper we present a fast algorithm for the computation of error-correcting graph isomorphisms. The new algorithm is an extension of a method for exact subgraph isomorphism detection from an input graph to a set of a priori known model graphs, which was previously developed by the authors.
\end{abstract}

\section{Introduction}

In pattern recognition and image analysis, graphs are often used for the representation of structured objects. If the problem is to recognize instances of known objects in an image, then often models of the known objects are represented by means of model graphs and stored in a database. The unknown objects in the input image are extracted by means of suitable preprocessing and segmentation algorithms, and represented by input graphs. Thus the problem of object recognition can be solved by searching for graph or subgraph isomorphisms between the models and the input graph. In a real world application, however, there is usually a certain amount of noise and distortion present in an input graph. Therefore, perfect correspondences between the models and the input do usually not exist. Hence, it is necessary to provide means for error-tolerant matching. In the past, different methods for finding exact and error-tolerant graph and subgraph isomorphisms have been proposed, such as heuristic search[BA83, SH81, Ul176], probabilistic relaxation[KCP92] or simulated annealing[HHVN90]. One of the major problems of error-correcting graph or subgraph isomorphism detection is its exponential time complexity, which is due to the fact that the problem is NP-complete. Combinatorial search methods such as $A^{*}$ are guaranteed to always find the optimal solution. However, they require exponential time in the worst case. Stochastic optimization methods such as relaxation or simulated annealing, on the other hand, have only polynomial time complexity, but they are not guaranteed to always yield the correct solution. Another problem with graph matching arises if the number of models in the database is large. In case of many models, it may become impossible to sequentially match each model against the input graph.

In this paper, we propose a method which is capable of finding all errorcorrecting graph isomorphisms between an input and a set of model graphs in time that is only polynomial in the number of vertices of the input graph. In particular, the time complexity of the new method is completely independent of 
the number of model graphs in the database. The new algorithm is an extension of the method for exact subgraph isomorphism detection that was previously presented by the authors in [MB95].

\section{Definitions and Notations}

Definition 1: A labeled graph $G$ is a 4-tuple, $G=(V, E, \mu, \nu)$, where (1) $V$ is the set of vertices, (2) $E \subseteq V \times V$ is the set of edges, (3) $\mu: V \rightarrow L_{V}$ is a function assigning labels to the vertices, (4) $\nu: E \rightarrow L_{E}$ is a function assigning labels to the edges.

In this definition, $L_{V}$ and $L_{E}$ are finite sets of symbolic labels. Let $G=(V, E, \mu, \nu)$ be a graph with $V=\left\{v_{1}, v_{2}, \ldots, v_{n}\right\}$. Then $G$ can also be represented by its adjacency matrix $M=\left(m_{i j}\right), i, j=1, \ldots, n$, where $m_{i i}=\mu\left(v_{i}\right)$ and $m_{i j}=\nu\left(\left(v_{i}, v_{j}\right)\right)$ for $i \neq j$. Clearly, the matrix $M$ is not unique for a graph $G$. If $M$ represents $G$, then any permutation of $M$ is also a valid representation of $G$.

Definition 2: A binary $n \times n$-matrix $P=\left(p_{i j}\right)$ is called a permutation matrix if the sum of the elements of each row and the sum of the elements of each column is equal to one.

If a graph $G$ is represented by an $n \times n$ adjacency matrix $M$ and $P$ is an $n \times n$ permutation matrix, then the $n \times n$ matrix

$$
M^{\prime}=P M P^{T}
$$

where $P^{T}$ denotes the transpose of $P$, is also an adjacency matrix of $G$. If $p_{i j}=1$ then the $j$-th vertex in $M$ becomes the $i$-th vertex in $M^{\prime}$.

Definition 3: Let $G_{1}$ and $G_{2}$ be two graphs and $M_{1}$ and $M_{2}$ their corresponding adjacency matrices. $G_{1}$ and $G_{2}$ are isomorphic if there exists a permutation matrix $P$ such that

$$
M_{2}=P M_{1} P^{T}
$$

Notice that the matrix $P$ can be understood as a bijective function $f$ that maps the vertices of $G_{1}$ to $G_{2}$, and vice versa. That is, $f\left(v_{j}\right)=v_{i}$ iff $p_{i j}=1$. We will call both $P$ and $f$ a graph isomorphism between $G_{1}$ and $G_{2}$. Thus, the problem of finding a graph isomorphism between two graphs $G_{1}$ and $G_{2}$ is equivalent to finding a permutation matrix $P$ for which Eq.(2) holds true.

In order to integrate the concept of error correction into graph matching, we define a distance measure for graphs which is based on the idea of correcting distortions in an input graph by means of edit operations [WF74, BA83]. The graph edit operations are used to modify either the model or the input graph until there exists a graph isomorphism between the model and the input. In order to model the fact that certain distortions, i. e. edit operations, are more 
likely than others, each graph edit operations $\delta$ is assigned a cost $C(\delta) \geq 0$. The graph distance from a model to an input graph is then defined to be the minimum cost taken over all sequences of edit operations that are necessary for the correction of the distortions in the input graph. In this paper, we consider the following distortions in a graph: vertex and edge label substitution, and missing and extraneous edges. For each type of distortion, a corresponding graph edit operation is defined.

Definition 4: Given a graph $G=(V, E, \mu, \nu)$, a graph edit operation $\delta$ on $G$ is any of the following:

$-\mu(v) \rightarrow l, v \in V, l \in L_{V}$ : substituting the label $\mu(v)$ of vertex $v$ by $l$ (for the correction of vertex label distortions).

$-\nu(e) \rightarrow l^{\prime}, e \in E, l^{\prime} \in L_{E}$ : substituting the label $\nu(e)$ of edge $e$ by $l^{\prime}$ (for the correction of edge label distortions).

- $e \rightarrow \$, e \in E$ : deleting the edge $e$ from $G$ (for the correction of missing edges).

- $\$ \rightarrow e=\left(v_{1}, v_{2}\right), v_{1}, v_{2} \in V,\left(v_{1}, v_{2}\right) \notin E$ : inserting an edge between two existing vertices $v_{1}, v_{2}$ of $G$ (for the correction of extraneous edges).

Definition 5: Given a graph $G=(V, E, \mu, \nu)$ and an edit operation $\delta$, the edited graph, $\delta(G)$, is a graph $\delta(G)=\left(V_{\delta}, E_{\delta}, \mu_{\delta}, \nu_{\delta}\right)$ with

1. $V_{\delta}=V$

2. $E_{\delta}= \begin{cases}E \cup\{e\} & \text { if } \delta=(\$ \rightarrow e) \\ E-\{e\} & \text { if } \delta=(e \rightarrow \$) \\ E & \text { otherwise }\end{cases}$

3. $\mu_{\delta}(v)= \begin{cases}l & \text { if } \delta=(\mu(v) \rightarrow l) \\ \mu(v) & \text { otherwise }\end{cases}$

4. $\nu_{\delta}(e)=\left\{\begin{array}{l}l^{\prime} \quad \text { if } \delta=\left(\nu(e) \rightarrow l^{\prime}\right) \\ \nu^{\prime}(e) \text { otherwise }\end{array}\right.$

$\nu(e)$ otherwise

Definition 6: Given a graph $G=(V, E, \mu, \nu)$ and a sequence of edit operations $\Delta=\left(\delta_{1}, \delta_{2}, \ldots, \delta_{k}\right), \quad k \geq 1$, the edited graph, $\Delta(G)$, is a graph $\left.\Delta(G)=\delta_{k}\left(\ldots \delta_{2}\left(\delta_{1}(G)\right)\right) \ldots\right)$. The total cost of the transformation of $G$ into $\Delta(G)$ is given by $C(\Delta)=\sum_{i=1}^{k} C\left(\delta_{i}\right)$.

Definition 7: Given two graphs $G$ and $G^{\prime}$, an error-correcting (ec) graph isomorphism from $G$ to $G^{\prime}$ is a 2-tuple $(\Delta, P)$ where $\Delta$ is a sequence of edit operations and $P$ is a graph isomorphism from $\Delta(G)$ to $G^{\prime}$. The cost of an $e c$ graph isomorphism $(\Delta, P)$ is the cost $C(\Delta)$.

It follows from this definition that, if there is an $e c$ graph isomorphism $(\Delta, P)$ from a graph $G$ to a graph $G^{\prime}$, then $G^{\prime}=P M_{\Delta(G)} P^{\prime}$ where $M_{\Delta(G)}$ is the adjacency matrix of the graph $\Delta(G)$. It is also easy to see that the permutation matrix $P$ is implied by $G, G^{\prime}$ and $\Delta$. 
Usually, there is more than one sequence $\Delta$ of edit operations such that a graph isomorphism from $\Delta(G)$ to $G^{\prime}$ exists. Consequently, there is usually more than one $e c$ graph isomorphism from $G$ to $G^{\prime}$. For our graph distance measure, we are particularly interested in the $e c$ graph isomorphism with minimum cost.

Definition 8: Let $G$ and $G^{\prime}$ be two graphs. The graph distance from $G$ to $G^{\prime}$, $d\left(G, G^{\prime}\right)$, is given by the minimum cost taken over all $e c$ graph isomorphisms from $G$ to $G^{\prime}$ :

$$
d\left(G, G^{\prime}\right)=M I N_{\Delta}\left\{C(\Delta) \mid(\Delta, P) \text { is an ec graph isomorphism from } G \text { to } G^{\prime}\right\}
$$

The $e c$ subgraph isomorphism $(\Delta, P)$ associated with $d\left(G, G^{\prime}\right)$ is called the optimal error-correcting (oec) graph isomorphism from $G$ to $G^{\prime}$. In the rest of this paper, we assume that the costs for substituting labels and for inserting and deleting edges are symmetric, i.e., $C\left(l_{1} \rightarrow l_{2}\right)=C\left(l_{2} \rightarrow l_{1}\right)$ for all labels $l_{1}, l_{2}$ and $C(e \rightarrow \$)=C(\$ \rightarrow e)$ for all edges $e^{1}$. It is easy to see that this assumption guarantees that $d\left(G, G^{\prime}\right)=d\left(G^{\prime}, G\right)$ for any pair of graphs, $G$ and $G^{\prime}$.

\section{Error-correcting Graph Isomorphism by Decision Tree}

Given a set of model graphs $G_{1}, \ldots, G_{L}$ and an input graph $G_{I}$ we want to find the oec graph isomorphism $\left(\Delta^{i}, P^{i}\right)$ between $G_{i}$ and $G_{I}$ such that the cost $C\left(\Delta^{i}\right)$ is minimal over all model graphs, i.e. $C\left(\Delta^{i}\right)=\min \left\{C\left(\Delta^{j}\right) ; j=1, \ldots, L\right\}$. Traditionally, this problem is solved by applying an $A^{*}$-based algorithm to each model-input graph pair [SH81, SF83, Won90, CYS $\left.{ }^{+} 96\right]$. Any such algorithm has an exponential time complexity in the worst case. Moreover, the method must be applied individually to each model-input pair. Consequently, the time complexity is also linearly dependent on the number of model graphs. In the case of exact graph isomorphism detection, both of these disadvantages can be avoided by using the decision tree approach described in [MB95]. Due to extensive preprocessing of the model graphs, the time complexity of this method is only polynomial in the number of nodes in the input graph. In particular, the time complexity is completely independent of the number and size of models in the database. For all further details, the reader is refered to [MB95].

The basic idea of applying the decision tree approach to error-correcting graph isomorphism detection is to separate the graph isomorphism search from the error-correction process. That is, given two graphs $G$ and $G^{\prime}$, we propose to generate all distorted copies of $G$ such that the graph distance from each copy to $G$ is not larger than a certain threshold $\vartheta$. Each of the distorted copies of $G$ is then separately matched with the graph $G^{\prime}$. Clearly, if the graph distance of $G$ and $G^{\prime}$ is not larger than $\vartheta$, there exists a distorted copy of $G$ in the generated

${ }^{1}$ This assumption is not essential, but it simplifies the description of the proposed method. 


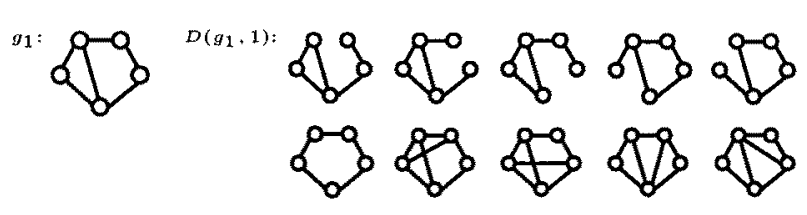

Fig. 1. The graph $g_{1}$ and the set $D\left(g_{1}, \vartheta\right)$ for $\vartheta=1$.

set that is isomorphic to $G^{\prime}$. Hence, this copy will be detected by the exact graph isomorphism process. Formally, let

$$
D(G, \vartheta)=\{\Delta(G) \mid \Delta \text { is a sequence of edit operations with } C(\Delta) \leq \vartheta\}
$$

denote the set of all edited copies of $G$ with cost less than or equal to $\vartheta$. Clearly, for any graph $G^{\prime}$, if $d\left(G, G^{\prime}\right) \leq \vartheta$, then $G^{\prime} \in D(G, \vartheta)$. An example is shown in Fig. 1. A graph $g_{1}$ and the set $D\left(g_{1}, \vartheta\right)$ for $\vartheta=1$ are displayed. As there are no labels the only possible edit operations are the insertion and the deletion of an edge. Each such operation is assigned a cost equal to 1 . Hence, $D\left(g_{1}, 1\right)$ consists of exactly ten edited copies of $g_{1}$.

After $D(G, \vartheta)$ has been computed, the optimal error-correcting graph isomorphism (if it exists) can be determined by testing each graph $G^{\prime \prime} \in D(G, \vartheta)$ with $G^{\prime}$ for graph isomorphism. There are two possible implementations of this idea. Given a model graph $G$ and an input graph $G_{I}$, we can either compute the set $D(G, \vartheta)$ or the set $D\left(G_{I}, \vartheta\right)$. In the first case, $D(G, \vartheta)$ can be computed off-line and at run time it is tested if $G_{I} \in D(G, \vartheta)$. In the second case, due to the fact that $G_{I}$ becomes available at run time only, $D\left(G_{I}, \vartheta\right)$ must be computed on-line before the condition $G \in D\left(G_{I}, \vartheta\right)$ can be tested. The first case is described in [MB96] in greater detail, while the second case will be discussed in this paper. In Fig. 2 the on-line error-correcting graph isomorphism algorithm based on a decision tree is outlined.

It can be shown that for $L$ model graphs with $n$ vertices each the size of the decision tree is $O\left(L 3^{n}\right)$ [MB96]. For a constant cost of 1 of each edit operation, the size of $D\left(G_{I}, \vartheta\right)$ for a graph $G_{I}$ with $n$ vertices is bounded by $O\left(\vartheta n^{2 \vartheta}\right)$. Therefore, the time complexity of the on-line $e c$ graph isomorphism algorithm based on a decision tree is bounded by $O\left(\vartheta n^{2(\vartheta+1)}\right)$. It is important to note that this time complexity is completely independent of the number of model graphs in the database. We conclude that the on-line error-correcting algorithm based on a decision tree is especially efficient if the database of model graphs is large and the maximal degree of distortion to be considered is rather small.

\section{Experimental Results}

In order to examine the efficiency of the new algorithm in practice, we have performed a number of experiments with randomly generated graphs. Both the new decision tree algorithm and a conventional, $A^{*}$-based algorithm were implemented in $\mathrm{C}++$ and run on a SUN Sparc10 Workstation. For each experiment, 
1. generate $D\left(G_{I}, \vartheta\right)$ by applying all edit operations and combinations of edit operations to $G_{I}$

2. for each $G_{I}^{\prime} \in D\left(G_{I}, \vartheta\right)$

(a) classify the adjacency matrix of $G_{I}^{\prime}$ with the decision tree representing the model graphs $G_{1}, \ldots, G_{L}$

(b) if $G_{I}^{\prime}$ is successfully classified by the decision tree as being isomorphic to the graph $G_{i}$, then add the error-correcting graph isomorphism between $G_{I}$ and $G_{i}$ to the list $F$.

3. output the error-correcting graph isomorphism with the least cost in $F$.

Fig. 2. Algorithm error-correcting_graph_isomorphism.

we generated one or more model graphs and used these model graphs to create input graphs that were distorted copies of the model graphs. All of the graphs generated for the experiments in this section were undirected and unlabeled. Each experiment was repeated 20 times and the average computation time was recorded. The size of the decision trees in terms of disk space is also given for each experiment. In the experiment described in the following, the performance of the on-line error-correcting algorithm was tested for varying model and database sizes and varying degrees of distortion.

In the 1st experiment, the size of the model graph was increased from 6 vertices and 12 edges to 16 vertices and 32 edges. The error threshold was kept at $\vartheta=1$. The computation time required by the new and by the conventional algorithm are displayed in Fig. 5. Notice that the decision tree method is much faster than the conventional algorithm. Graphs with up to 16 vertices can be easily handled. In the 2 nd experiment, the error threshold was set to $\vartheta=2$. The size of the model graphs was again increased from 6 vertices and 12 edges to 16 vertices and 32edges. The results of this experiment are displayed in Fig. 6 . Note that for graphs with less than 14 vertices, the conventional algorithm is faster than the decision tree approach. For larger graphs, however, the decision tree approach outperforms the conventional algorithm.

In the 3rd and the 4th experiment, we tested the performance of the on-line decision tree approach for a growing number of model graphs. In both experiments, the number of models in the database was gradually increased starting at one and ending at five graphs. Each model graphs consisted of 11 vertices and 22 edges. In the 3rd experiment documented in Fig. 5, the error threshold was kept at $\vartheta=1$ while in the 4th experiment documented in Fig. 6, the error threshold was set to $\vartheta=2$. Notice that in both experiments, the time required by the decision tree method was independent of the number of model graphs while the conventional method's performance was linearly dependent on the size of the 


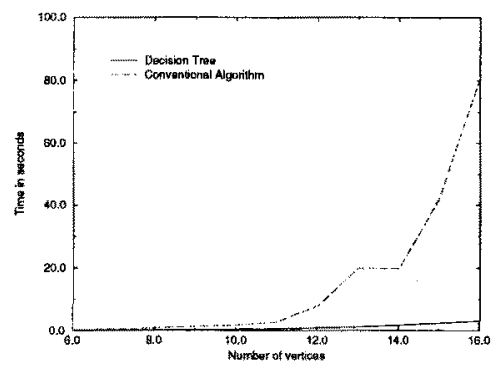

Fig. 3. Computation time in seconds for $\vartheta=1$ and a growing number of vertices (1st experiment).

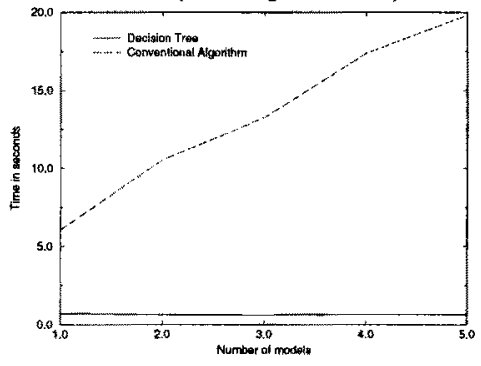

Fig. 5. Computation time in seconds for $\vartheta=1$ and a growing number of models (3rd experiment).

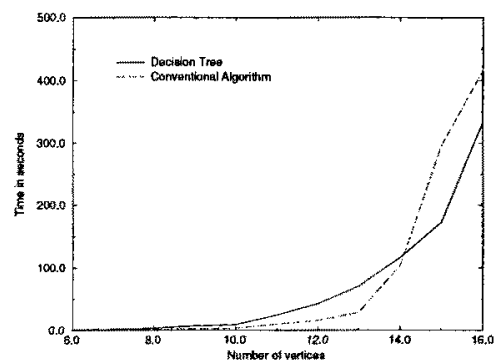

Fig. 4. Computation time in seconds for $\vartheta=2$ and a growing number of vertices (2nd experiment).

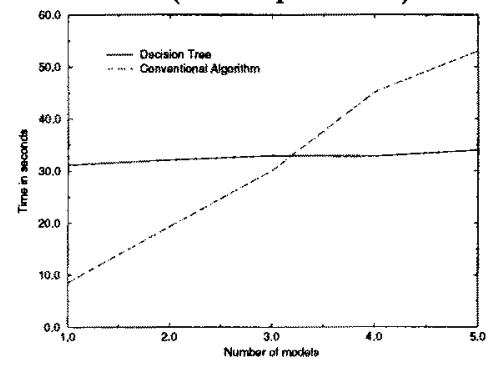

Fig. 6. Computation time in seconds for $\vartheta=2$ and a growing number of models (4th experiment).

database. We can observe that for $\vartheta=1$ the decision tree approach is always faster than the conventional algorithm. For $\vartheta=2$, the decision tree approach is slower than the conventional algorithm when the database is small. But for more than 3 models in the database, the decision tree method becomes superior.

\section{Conclusions}

We have presented a new algorithm for the problem of error-correcting graph isomorphism detection based on the decision tree paradigm. The new algorithm is an extension of an algorithm for exact graph and subgraph isomorphism detection that was developed by the authors before [MB95]. Its time complexity is quadratic in the size of the model graphs and exponential in the error threshold that is to be considered. Very important is the fact that the method is completely independent of the number of model graphs that are represented by the decision tree. On the other hand, the size of the decision tree grows exponentially with the size of the model graphs for both new algorithms.

The results of the theoretical complexity analysis have been confirmed in a number of practical experiments with randomly generated graphs. The advantages of the new algorithm in terms of computational performance were demonstrated in these experiments for graphs with up to 16 vertices. 


\section{Acknowledgment}

This work has been part of a project of the Priority Program SPP IF, No: 5003-34285, funded by the Swiss National Science Foundation.

\section{References}

[BA83] H. Bunke and G. Allerman. Inexact graph matching for structural pattern recognition. Pattern Recognition Letters 1, 4:245-253, 1983.

[CYS ${ }^{+96]}$ L. Cinque, D. Yasuda, L.G. Shapiro, S. Tanimoto, and B. Allen. An inproved algorithm for relational distance graph matching. Pattern Recognition, 29(2):349-359, 1996.

[HHVN90] L. Herault, R. Horaud, F. Veillon, and J.J. Niez. Symbolic image matching by simulated annealing. In Proc. British Machine Vision Conference, pages 319-324. Oxford, 1990.

[KCP92] J. Kittler, W. J. Christmas, and M. Petrou. Probabilistic relaxation for matching of symbolic structures. In H. Bunke, editor, Advances in Structural and Syntactic Pattern Recognition, pages 471-480. World Scientific, 1992.

[MB95] B.T. Messmer and H. Bunke. Subgraph isomorphism detection in polynomial time on preprocessed model graphs. In Proceedings of the Asian Conference on Computer Vision ACCV, pages 151-155, 1995.

[MB96] B.T. Messmer and H. Bunke. Fast error-correcting graph isomorphism based on model precompilation. Technical Report IAM-96-012, University of Bern, 1996.

[SF83] A. Sanfeliu and K.S. Fu. A distance measure between attributed relational graphs for pattern recognition. IEEE Transactions on Systems, Man, and Cybernetics, 13:353-363, 1983.

[SH81] L.G. Shapiro and R.M. Haralick. Structural descriptions and inexact matching. IEEE Transactions on Pattern Analysis and Machine Intelligence PAMI, 3:504-519, 1981.

[Ull76] J.R. Ullman. An algorithm for subgraph isomorphism. Journal of the Association for Computing Machinery, 23(1):31-42, 1976.

[WF74] R.A. Wagner and M.J. Fischer. The string-to-string correction problem. Journal of the Association for Computing Machinery, 21(1):168-173, 1974.

[Won90] E. K. Wong. Three-dimensional object recognition by attributed graphs. In H. Bunke and A. Sanfeliu, editors, Syntactic and Structural Pattern Recognition- Theory and Applications, pages 381-414. World Scientific, 1990. 